\title{
Signal integration: a framework for understanding the efficacy of therapeutics targeting the human EGFR family
}

\author{
H. Michael Shepard, ${ }^{1}$ Cathleen M. Brdlik, ${ }^{1}$ and Hans Schreiber ${ }^{2}$
}

${ }^{1}$ Receptor BioLogix, Inc., Palo Alto, California, USA. ${ }^{2}$ Department of Pathology, University of Chicago, Chicago, Illinois, USA.

\begin{abstract}
The human EGFR (HER) family is essential for communication between many epithelial cancer cell types and the tumor microenvironment. Therapeutics targeting the HER family have demonstrated clinical success in the treatment of diverse epithelial cancers. Here we propose that the success of HER family-targeted monoclonal antibodies in cancer results from their ability to interfere with HER family consolidation of signals initiated by a multitude of other receptor systems. Ligand/receptor systems that initiate these signals include cytokine receptors, chemokine receptors, TLRs, GPCRs, and integrins. We further extrapolate that improvements in cancer therapeutics targeting the HER family are likely to incorporate mechanisms that block or reverse stromal support of malignant progression by isolating the HER family from autocrine and stromal influences.
\end{abstract}

\section{Introduction}

A solid tumor is composed of cancer cells embedded in abundant stroma consisting of nontransformed stromal cells and ECM (in this article we use "tumor" to refer to the mass of cancer cells together with nonmalignant stromal cells) (1). It is now widely accepted that within this specialized microenvironment, there is a complex interplay between the cancer cells and the stroma, which strongly influences the development, progression, and metastatic potential of the cancer cells $(2,3)$. One of the earliest recognized properties of cancer cells is their growth factor self-sufficiency, which is achieved by activation of cellular proto-oncogenes, by either mutation or overexpression (4-6). Further evidence of the importance of growth factors (a subclass of cytokines) and their receptors in tumor progression includes the association between cancer and aberrant signal transduction mediated by growth factor receptors, such as members of the human EGFR (HER) family $(7,8)$. Indeed, activation of HER family members leads not only to increased cell proliferation, but also to cancer cell resistance to growth-inhibitory cytokines and expression of selective immunosuppressive and proangiogenic cytokines and chemokines $(9,10)$, thereby creating an environment that favors tumor progression.

Therapeutic reagents targeting HER family members, in particular EGFR (also known as HER1) and the receptor tyrosine kinase (RTK) encoded by HER2, p185 HER2 (also known as HER2, HER2/neu, and ErbB2), have proven successful for the treatment of breast, colon, lung, and pancreatic cancers $(11,12)$. However, not all tumors expected to respond to these therapeutics are in fact sensitive to them, and in many cases resistance eventually develops. We believe that broader success in developing therapies directed at HER family members depends upon an increased appreciation for how these receptors consolidate signals from diverse sources and

Nonstandard abbreviations used: ADAM, a disintegrin and metalloprotease; ADCC, antibody-dependent immune cell killing; HER, human EGFR; LPA, lysophosphatidic acid; NRG, neuregulin; RTK, receptor tyrosine kinase; TKI, tyrosine kinase inhibitor.

Conflict of interest: H.M. Shepard and C.M. Brdlik are employees of Receptor BioLogix, Inc., which is engaged in the development of multimeric decoy receptors derived from the human EGFR family.

Citation for this article: J. Clin. Invest. 118:3574-3581 (2008). doi:10.1172/JCI36049. how they cooperate toward functional homeostasis in cancer cells. This article discusses a diverse body of work that we hope provides a basis for the design of new therapeutic approaches to cancers associated with aberrant activation of HER family members.

\section{The HER family and cancer}

The HER family is composed of four receptors (Figure 1), plus a number of variants generated by alternative splicing, and 11 types of ligands, some of which are also generated by alternative splicing (e.g., the neuregulins [NRGs]) (13). Roles for these receptors and their ligands have been described in many types of human cancer, including breast, colon, pancreatic, ovarian, brain, and lung cancers (14). While appropriate focus has been on malignant cell expression of HER family members, expression of EGFR and TGF- $\alpha$ on tumor-associated stromal endothelial cells has also been reported and could be of therapeutic significance (15).

HER family members are activated through oligomerization, and the minimal active configuration is either a homodimer or a heterodimer (Figure 1) (16). Activation can be achieved by ligand binding. This is true even for $\mathrm{p} 185^{\mathrm{HER} 2}$, which has no known soluble ligands and acts as a coreceptor for the other HER family members. In addition, constitutive activation can occur in the absence of endogenously added ligand if either EGFR or $\mathrm{p} 185^{\mathrm{HER} 2}$ is overexpressed or if activating mutations occur in their tyrosine kinase domain (17-19). This constitutive activity can be increased if cells are treated with HER family ligands $(20,21)$. In addition, there are other proteins on the cell surface that may enhance the ability of $\mathrm{p} 185^{\mathrm{HER} 2}$ to associate with other HER family receptors or to signal constitutively when it is overexpressed.

The contribution of each HER family member to cancer cell behavior is generally regulated by a combination of its level of expression and the availability of its cognate ligand(s) in the tumor microenvironment. Overexpression of single HER family members and their ligands is now known to predict more aggressive behavior of many epithelial malignancies (including breast cancer, non-small cell lung cancer, and bladder cancer) (13, 14, 22). Furthermore, the idea that cooperativity among HER family members creates a malignant phenotype is strongly supported by mechanistic and other nonclinical data. One fundamental 


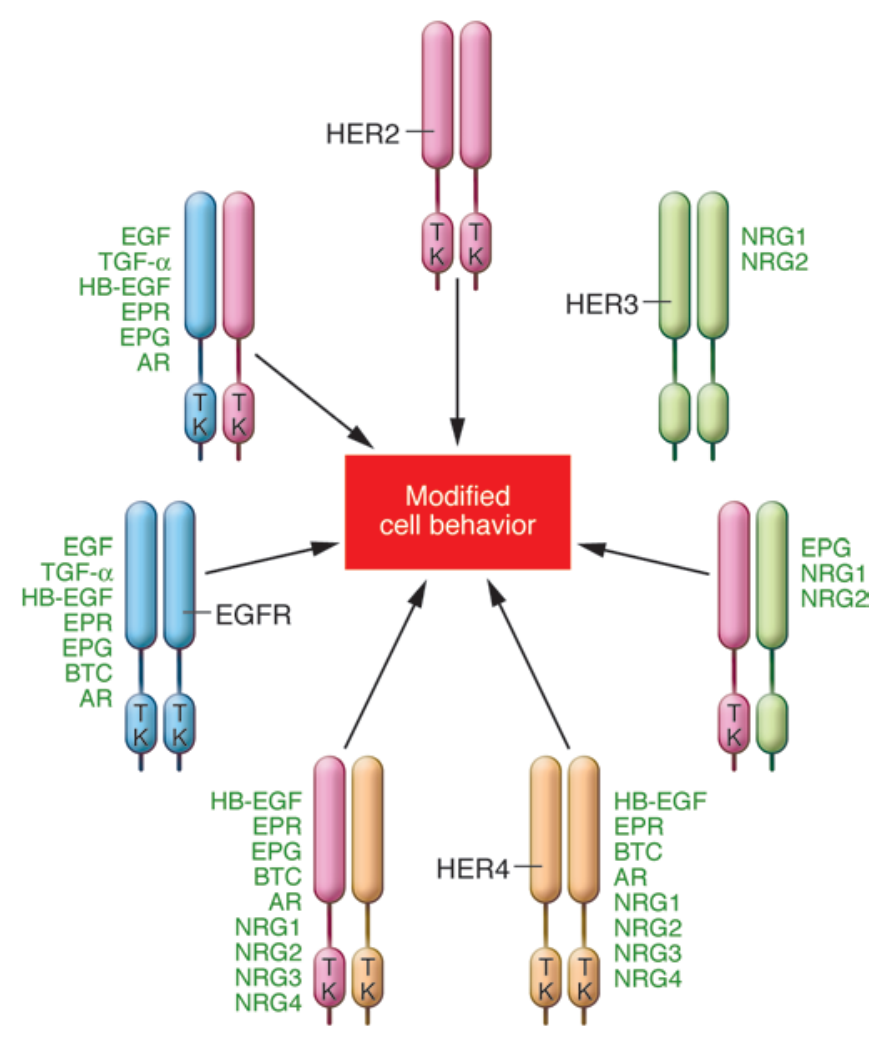

observation that is demonstrative of HER family cooperativity is that HER3 does not have a functional tyrosine kinase domain but rather depends upon NRG binding (Figure 1) and subsequent heterodimer formation, in particular with $\mathrm{p} 185^{\mathrm{HER} 2}$, in order to be transphosphorylated and achieve signaling competence (23). Other work has proven that coexpression of HER family members and their ligands synergizes for cellular transformation in vitro $(24,25)$ and that coexpression of HER family members can activate cell signaling and tumor cell invasion pathways not activated by single receptors (26). Other data further emphasize the complex cooperative nature of the HER family, not only with each other, but also with other inflammatory proteins, in driving tumor progression. For instance, overexpression of $\mathrm{p} 185^{\mathrm{HER} 2}$ in breast cancer cells is associated with enhanced expression of the angiogenic proteins IL- 8 and $\operatorname{VEGF}(9,10)$. Similarly, both overexpression of EGFR and activating mutations in the tyrosine kinase domain of EGFR induce cytokine production (in particular, VEGF, IL-6, and IL-8) in animal models of human cancer and in primary human lung adenocarcinomas (27-29). Other recent results have demonstrated a tyrosine kinase-independent role for EGFR in preventing autophagic cell death by maintaining intracellular glucose levels through interaction with the sodium/ glucose transporter SGLT-1 (30). Taken together, these data demonstrate cooperativity among HER family members, not just in the malignant behavior of cancer cells, but also in modifying the tumor microenvironment to favor tumor progression.

A significant amount of literature relating to prognostic outcome and HER family coexpression supports the concept that more successful therapeutics will require simultaneous suppression of the aberrant activation of multiple HER family members. Most importantly, analysis of patient samples has shown that patients whose cancers are characterized by the coexpression of

\section{Figure 1}

The HER family and its ligands. There are four members of the HER family: EGFR (also known as HER1), HER2, HER3, and HER4. EGFR, HER2, and HER4 have a cytoplasmic tyrosine kinase (TK) domain that is activated by oligomerization. The HER3 kinase domain differs due to a lack of TK function. The minimal active configuration is either a homodimer or a heterodimer. HER family oligomerization is usually the result of binding by one of 11 types of ligand. This is even true for HER2, which is a coreceptor without a described soluble ligand. Oligomerization can also occur if either EGFR or HER2 is overexpressed or if activating mutations occur in their TK domain. Additional complexity is inherent in the HER system, since each receptor and many of the ligands can be products of alternative splicing, resulting in amplification of functional diversity. AR, amphiregulin; BTC, betacellulin; EPG, epigen; EPR, epiregulin; HB-EGF, heparin-binding EGF.

two or more HER family members demonstrate a more aggressive malignancy than is observed when only one member is expressed in the cancer cells (31-35). In one study (35), patients with breast cancer expressing a single HER family member had a median tenyear survival rate of $75 \%$, as compared with $40 \%$ for patients whose cancer expressed two or more HER family members.

\section{Link between TNF- $\alpha$, incipient tumor progression, and the HER family: the conception of trastuzumab}

Incipient cancer cells progress toward malignancy as a result of epigenetic and genetic changes coupled with continuous in vivo selection for the most malignant cells $(5,36)$. One mechanism of host protection against incipient tumor progression involves the production of TNF- $\alpha$ by immune cells in the stroma as part of the antitumor innate immune response $(36,37)$. Overcoming this component of host immunosurveillance, for example by selecting for resistance to TNF- $\alpha$ in vitro, can convert a regressing syngeneic mouse tumor into a progressively growing lesion (38). This phenomenon is also relevant to human cancer cells: an extensive screen of human cancer cell lines surprisingly demonstrated that most are inherently resistant to TNF- $\alpha$-induced cytotoxicity (39). Investigations into the specific mechanisms that give rise to the TNF- $\alpha$-resistant phenotype were initiated with the goal of exploiting defined resistance mechanisms as targets for the development of novel therapeutics. These experiments showed that cancer cell resistance to the cytotoxic effects of TNF- $\alpha$ could be induced by the EGFR ligands EGF and TGF- $\alpha$, as well as other growth factors (40). The ability of growth factors to inhibit TNF- $\alpha$-mediated cytotoxicity suggested that one mechanism by which cancer cells could escape immunosurveillance is through autocrine or stromal cell production of growth factors.

The overexpression of $\mathrm{p} 185^{\mathrm{HER} 2}$ became the focus of work related to the mechanisms of cancer cell resistance to TNF- $\alpha$ when experiments showed that ligand (EGF or TGF- $\alpha$ ) activation of EGFR resulted in diminished TNF- $\alpha$-mediated cytotoxicity (40). Simultaneously, it was also shown that overexpression of p185 ${ }^{\text {HER2 }}$ in NIH 3 T3 fibroblasts resulted in oncogenic transformation (41), without the accompanying mutations reported for transformation of NIH $3 \mathrm{~T} 3$ cells by the NEU oncogene (42). In addition, soon after its initial characterization (43-45), amplification/overexpression of HER2 was linked to aggressive breast cancer and other malignancies (46-49). Subsequent studies tested whether overexpression of $\mathrm{p} 185^{\mathrm{HER} 2}$ was associated with cancer cell resistance to TNF- $\alpha$. In vitro analysis of NIH 3T3 fibroblasts transformed by overexpression of $\mathrm{p} 185^{\mathrm{HER} 2}$ demonstrated that these 
cells were resistant both to TNF- $\alpha$ and to cytotoxicity mediated by LPS/IFN- $\gamma$-activated macrophages (50), which use TNF- $\alpha$ as a major mechanism of immunosurveillance against incipient cancer cells (38). Similarly, increased resistance to the cytotoxic effects of TNF- $\alpha$ is demonstrated by breast tumor cells that express elevated p185 ${ }^{\text {HER2 }}$-associated tyrosine kinase activity (50). Further experiments demonstrated that TNF- $\alpha$ resistance of transformed cells in vitro was associated with an increased rate of dissociation of TNF- $\alpha$ from its receptor (50). More recently, cancer cell resistance to TNF- $\alpha$ has been associated with upregulation of cell survival pathways involving Akt and NF- $\mathrm{\kappa B}(51,52)$. Overexpression of p185 ${ }^{\text {HER2 }}$ has also been shown to interfere with the antiproliferative activity of IFN- $\gamma$ and TGF- $\beta$, both of which may be important for inhibition of incipient tumor progression $(2,37,53,54)$.

These initial findings stimulated a search for an antagonistic $\mathrm{mAb}$ that could recognize the extracellular domain of $\mathrm{p} 185^{\mathrm{HER} 2}$ and inhibit cancer cell growth while enhancing the sensitivity of HER2overexpressing cancer cells to TNF- $\alpha$. Of the more than $100 \mathrm{mAbs}$ derived as specific for the extracellular domain of p185 HER2 (55) and studied in detail (56), the antibody designated muMAb4D5 demonstrated the highest degree of correlation between $\mathrm{p} 185^{\text {HER2 }}$ expression and growth inhibition of both breast cancer cell lines in vitro and human breast cancer xenografts in nude mice (56-58). Treatment with muMAb4D5 was also shown to convert TNF- $\alpha-$ induced growth inhibition to a cytotoxic response in HER2overexpressing breast cancer cells in vitro, but it did not have this effect on cancer cell lines that did not overexpress HER2 (57). The link between TNF- $\alpha$ resistance and proto-oncogene overexpression was further substantiated by subsequent work showing that selection of NIH $3 \mathrm{~T} 3$ fibroblasts for resistance to the cytotoxic effects of TNF- $\alpha$ leads to enrichment in the remaining cells for those with a transformed morphology and is often associated with amplified copy number and overexpression of the c-MET proto-oncogene, which encodes hepatocyte growth factor receptor (59). It is interesting to speculate that some incipient cancer cells might escape immune cells secreting TNF- $\alpha$ in vivo, as part of the antitumor innate immune response, leading to the formation of tumors characterized by aberrant constitutive RTK activation or RTK overexpression.

The demonstration that muMAb4D5 inhibited tumor cell growth in vitro in a manner that correlated with overexpression of HER2, that it induced a cytotoxic response to TNF- $\alpha$ in vitro (also specific for HER2-overexpressing tumor cells), and that it inhibited the growth of HER2-overexpressing human breast cancer in xenograft models (58) led to the development of humanized muMAb4D5 (trastuzumab) and its subsequent application in breast cancer. Trastuzumab was designed as an IgG1 subtype to provide the potential for antibody-dependent immune cell killing (ADCC) of breast cancer cells that overexpress p185 HER2 (56, 60). This aspect of trastuzumab might be especially relevant for its clinical effects, as, when overexpressed, $\mathrm{p} 185^{\mathrm{HER} 2}$ tends to cluster with about $10^{3}$ receptors per cluster in SK-BR-3 tumor cells in culture, an effect that is potentiated by trastuzumab (61-63). Following binding to cell surface $\mathrm{p} 185^{\mathrm{HER} 2}$, trastuzumab can subsequently couple to and induce clustering of Fc receptors (FcrRIIIa) on stromal immune cells, especially NK cells, resulting in tumor cell killing mediated by release of perforin, granzyme, and cytokines (64). The activation of ADCC enables a mechanism for antitumor activity of trastuzumab that is independent of its other mechanisms of action and that results in preferential tar- geting of immune cells to HER2-overexpressing tumor cells in vitro (56). The activation of ADCC may enable a mechanism for the antitumor activity of trastuzumab that is independent of its other mechanisms of action and that allows preferential targeting of immune cells to $185^{\mathrm{HER} 2}$-overexpressing cancers $(56,65)$. Because HER2 expression persists in trastuzumab-resistant tumor cells (66), ADCC tumor cell cytotoxicity may provide an independent mechanism for efficacy mediated by trastuzumab (67).

Trastuzumab therefore serves as a model for the development of therapeutics that can simultaneously target malignant cell behavior and interactions between stromal cells and cancer cells. Trastuzumab directly inhibits breast cancer cell proliferation by disrupting the function of $\mathrm{p} 185^{\mathrm{HER} 2}$ (58). Other consequences of trastuzumab treatment of HER2-overexpressing breast tumor cells include: increased sensitivity to growth inhibition by the cytokine TNF- $\alpha$ (57); downregulation of tumor cell production of IL-8, an inflammatory chemokine with proangiogenic activity (10); downregulation of tumor cell VEGF production (10); and downregulation of CXCR4, the receptor for CXCL12 (also known as stromalderived growth factor 1). Downregulation of CXCR4 may inhibit metastasis of breast cancers that overexpress p185 ${ }^{\text {HER2 }}$ (68). These results suggest that overexpression of $\mathrm{p} 185^{\mathrm{HER} 2}$ plays a central role in breast cancer tumor progression by inducing the expression of multiple protumorigenic signals and that trastuzumab exerts its efficacy by multiple mechanisms that depend on downregulating expression of $\mathrm{p} 185^{\mathrm{HER} 2}(21,58)$.

Several conclusions can be drawn from the results discussed to this point. The constitutive activation of a single HER family member creates more aggressive malignancy, and cooperation among HER family members enhances this effect. The mechanisms that contribute to more aggressive malignancy include increased tumor cell proliferation, as well as HER-associated tumor cell resistance to the innate immune system (including the growth inhibitory cytokines TNF- $\alpha$, TGF- $\beta$, and IFN- $\gamma$ ). Last, as described earlier, aberrant activation of HER family members induces cancer cell production of cytokines and chemokines that impact stroma and encourage tumor progression. $\mathrm{mAb}$ antagonists that target HER family members, in particular trastuzumab (which targets p185 HER2) and cetuximab (which targets EGFR), are successful therapeutics. A possible framework to explain the success of these therapeutics is that the HER family integrates signals from a multitude of other receptor systems, many of which are involved in cytokine and chemokine signaling, and that trastuzumab and cetuximab interfere with both the response of the tumor cell to these signals and the ability of the tumor cell to produce protumorigenic cytokines that activate the stroma.

\section{Signal integration by the HER family}

Transactivation of the HER family occurs when the cytoplasmic domain of one or more family members becomes tyrosine phosphorylated and signaling competent following an event initiated by a distinct "upstream" receptor family (Figure 2). As discussed here, we believe that this places the HER family at the center of converging signals for cell proliferation, motility, and other cancer cell behaviors.

The accumulated evidence supports a model in which HER family transactivation and cooperation represents a primary driver of HER function and subsequent modification of cancer cell behavior (Figure 2), as suggested earlier by Hynes and Britten $(69,70)$. The most common mechanism of HER transactivation is through 


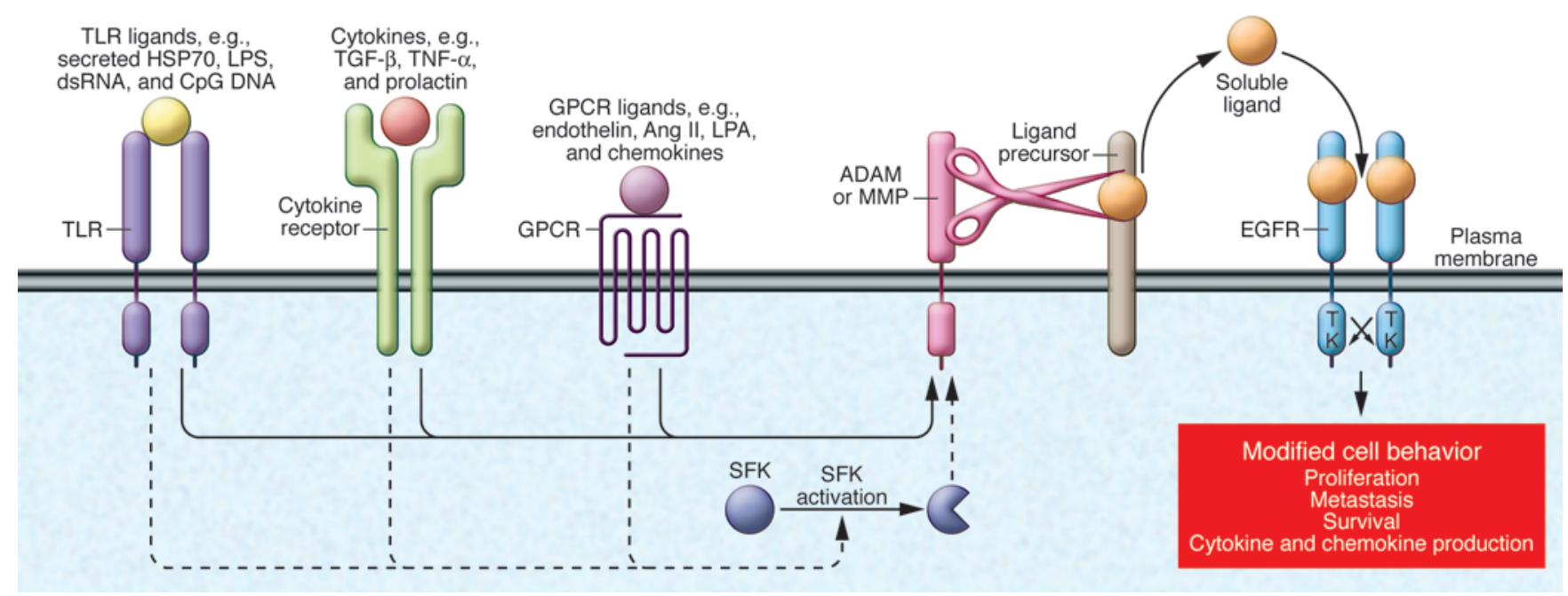

Figure 2

Members of the HER family consolidate signals from cancer and stromal cells. There are a multitude of signaling molecules secreted by cancer cells and the tumor-associated stromal cells (e.g., inflammatory cells, endothelial cells, and fibroblasts) that modulate HER family function. Activation of GPCRs (e.g., by chemokines), TLRs (e.g., by HSP70), and cytokine receptors (e.g., by TGF- $\beta$ ) can lead to transactivation of HER family members, as shown here for EGFR. These receptors link the HER family to innate and adaptive immunity, including immunosurveillance, which is important for protection against incipient tumor progression (38). Although not shown here, activation of other receptors, e.g., receptors for WNT (116) and integrins, can also lead to transactivation (117). Although only transactivation of EGFR is shown in this figure, other HER family members are also subject to transactivation by the same diversity of ligand/receptor systems. Transactivation is mediated primarily by metalloproteinase activation (ADAMs and MMPs) of precursor HER ligands and also by intracellular signal transducers (such as Src family kinases [SFKs]), which can amplify this effect. This creates a situation in which the HER family acts as a conduit for diverse signaling systems. Most of the receptor systems described here are present in both nonmalignant stromal cells and cancer cells.

upstream activation of GPCRs by their requisite ligands (71-73), including lysophospholipids, oligopeptide hormones (such as angiotensin II), and chemokines (74-77), followed by metalloproteinase-catalyzed release of HER ligands. In the best-characterized examples, GPCR activation results in G-protein-mediated activation of ADAM (a disintegrin and metalloprotease) family members, which then cleave HER pro-ligands. The mature HER ligands that are subsequently released activate their corresponding receptors on cancer or stromal cells, resulting in $\operatorname{HER}$ activation $(71,73,78)$. In addition to GPCRs, many other ligand/receptor systems have been documented by several groups to elicit transactivation of the HER family, with most studies focusing on EGFR (Figure 2) (70, 73). Examples include cytokine receptors (79-81) and TLRs (Figure 2). TLRs are classically activated by pathogen-derived ligands and are primarily associated with the innate immune response (chemokine and cytokine release). Importantly, TLRs can also be activated as a result of release of malignant cell contents (such as HSP70; Figure 2) $(82,83)$, which occurs frequently in progressing cancer cells (37). Although the main objective of this article is to discuss the central role of the HER family in malignancy, it should be appreciated that the HER family can play a role in pathologies as diverse as rheumatoid arthritis (84) and schizophrenia (13).

The receptor/ligand systems that can transactivate the HER family via maturation of proligands are numerous and diverse and may be found to involve many of the more than 800 described GPCRs. These diverse receptors can also transactivate the HER family by other mechanisms (73), primarily via Src family kinases (SFKs; Figure 2). In the case of EGFR, c-Src appears to functionally synergize with EGFR ligand-mediated activation through a mechanism that includes colocalization of c-Src and ligand-acti- vated EGFR in endosomes (85). This function of c-Src maximizes the effects of ligand activation. Although most of the data discussed above are derived from studies of EGFR, transactivation has also been reported for other members of the HER family (86-88). We propose here that signal consolidation by the HER family is important to its role in disease progression and represents part of the explanation for the success of therapeutics that target these receptors.

\section{The predominant mechanisms of resistance to mAbs targeting HER family members}

Except for dominant signal pathway mutations, such as functional loss of the phosphatase and tensin homolog tumor suppressor gene (PTEN) (89), the mechanisms of inherent and acquired resistance to mAbs targeting HER family members have been difficult to define. One method to test whether HER family members cooperate to have an impact on cancer cell sensitivity to monotherapy with a mAb targeted to a single HER family member (e.g., resistance to the EGFR-targeted mAb cetuximab) is to utilize the p185 18 HER-directed mAb $2 \mathrm{C} 4(56,58)$, which binds to p185 ${ }^{\text {HER2 }}$ and interferes with some HER family dimerization pathways (90). Because dimerization is required for $\mathrm{p} 185^{\mathrm{HER} 2}$ signaling, and $\mathrm{p} 185^{\mathrm{HER} 2}$ is the preferred dimerization partner for other HER family members $(16,91)$, it has been proposed that $2 \mathrm{C} 4$, or its humanized counterpart pertuzumab, should broadly disrupt HER family associations. Support for this point of view has been provided by the demonstration that pertuzumab treatment can reverse acquired resistance to cetuximab in vitro (92). This is presumably because it functionally disrupts HER2 interactions with the EGFR and other HER family members (93). 


\begin{abstract}
Table 1
Inhibition of LPA-stimulated proliferation of tumor cells in vitro by RB200
\end{abstract}

$\begin{array}{lcc}\text { Tumor cell line } & \text { Tumor type } & \begin{array}{c}\text { Inhibition of LPA-induced } \\ \text { proliferation by RB200 }\end{array} \\ \text { H1975 } & \text { Lung } & ++++ \\ \text { H441 } & \text { Lung } & +++ \\ \text { H1993 } & \text { Lung } & +++ \\ \text { A549 } & \text { Lung } & +++ \\ \text { Calu-1 } & \text { Lung } & +++ \\ \text { H358 } & \text { Lung } & ++ \\ \text { HCC4006 } & \text { Lung } & + \\ \text { H2122 } & \text { Lung } & - \\ \text { A431 } & \text { Epidermoid } & ++ \\ \text { MCF-7 } & \text { Breast } & ++++ \\ \text { MDA-MB-231 } & \text { Breast } & - \\ \text { N-87 } & \text { Gastric } & ++ \\ \text { HT-29 } & \text { Colon } & -\end{array}$

Assays were performed as described in ref. 21 in serum-free medium with or without $5.0 \mu \mathrm{M}$ LPA and with or without RB200 (EGFR:HER3/Fc bispecific ligand trap). Inhibition of GPCR ligand-stimulated (LPA) proliferation by RB200:,$-<10 \% ;+, 10 \%-20 \% ;++, 21 \%-50 \% ;+++$, $51 \%-70 \% ;++++,>70 \%(21)$.

Important support for HER family cooperation in resistance to monotherapy with a mAb targeted to a single HER family member is provided by the demonstration that resistance to the HER2-targeted mAb trastuzumab in HER2-overexpressing BT474 breast tumors, selected in vivo, is associated with increased expression of EGFR, increased numbers of EGFR-HER2 heterodimers, and increased levels of mRNAs encoding TGF- $\alpha$, heparin-binding EGF (HB-EGF), and NRG (66). In addition, simultaneous $m A b$ targeting of multiple epitopes and members of the HER family has demonstrated synergistic antiproliferative effects on cancer cells in vitro (61). Thus, cooperation among HER family members and their associated growth factors appears to be a major culprit in acquired resistance to $\mathrm{mAb}$ therapeutics targeting the $\operatorname{HER}$ family $(66,92,94,95)$.

\section{The current challenge}

Trastuzumab and cetuximab are groundbreaking anticancer therapeutics, but they are active in only a fraction of their targeted patient populations $(11,12)$. Resistance to trastuzumab monotherapy and trastuzumab in combination with chemotherapy is multifactorial, but we believe that the best-documented association with acquired resistance is the upregulation and/or increased association of the therapeutically targeted receptor with other nontargeted members of the HER family $(23,66,92,95)$. This conclusion is supported by the discussion above and by Arpino et al. (96), who used two xenograft models of HER2-overexpressing breast cancer to show that a combination of gefitinib (a nonproteinaceous tyrosine kinase inhibitor [TKI] specific for the EGFR), trastuzumab, and pertuzumab (which targets a distinct HER2 epitope to trastuzumab) (58, 97) provided significantly improved antitumor efficacy compared with any single agent and any dual combination of the agents. These results provide a valuable rationale for developing new therapeutics that can more broadly interfere with HER family function.

Clinical efforts for the purpose of developing mAbs targeting multiple members of the HER family ("pan-HER" approaches) have been focused on $\mathrm{p} 185^{\text {HER } 2}$, because of its central role as a driver for HER family signaling in HER2-overexpressing breast cancer and its proposed role as a signaling amplifier for other HER family members (98). So far, these efforts have yielded limited success $(99,100)$. A possible explanation is that HER2 may not play as dominant a role in most cancers as it does in HER2-overexpressing breast cancers. Instead, in many malignancies, HER2 may function as a coreceptor, responding primarily to activation by other HER family members. Different approaches are therefore needed and should take into account the multiplicity of ligands that activate the HER family (Figure 1) as well as the cooperative and self-compensating behavior of the receptors themselves (95). Treatment with a mixture of $\mathrm{mAbs}$ and TKIs (including SFK inhibitors) that simultaneously target multiple receptor epitopes and tyrosine kinases has shown promise in a number of preclinical models $(96,101)$ and is one approach currently undergoing clinical evaluation (102).

A second pan-HER approach targets the ADAM17 metalloproteinase, which is important in HER ligand maturation (Figure 2) (103). Because soluble growth factors driving HER family transactivation originate in a metalloproteinase-dependent manner from both cancer cells and nonmalignant stromal cells, an ADAM17 antagonist might provide a means of therapeutically targeting both cancer cells and hyperactivated stromal cells to downregulate HER family function. Some disadvantages of this approach are that previous attempts to inhibit metalloproteinases resulted in off-target activity (104) and that the ADAM family exhibits tremendous redundancy (105).

An approach with the inherent specificity of a biologic therapeutic is "ligand trap" technology, first proven successful with etanercept, a fusion protein comprising the extracellular domain of human p75-TNFR linked to the Fc component of human IgG1 (IgG1Fc) (106). To create a pan-HER ligand trap, the ligand binding domains of EGFR and HER3 have been combined into a human IgG1Fc-mediated heterodimeric configuration designated as RB200 (21). EGFR was chosen as one arm of the ligand trap in order to sequester most growth factors that activate the HER family (7 of the 11 ligands of HER family members bind EGFR) (Figure 1). HER3 was chosen as the second subunit because its activation appears to have an important role in resistance to HER-targeted therapeutics $(23,92,95)$. Blocking ligandmodulated activation in this way might isolate cancer cells from stromal or autocrine growth factors, the protumorigenic effects of which are consolidated through the HER family. Because of the importance of transactivation by GPCRs in activating the HER family, initial in vitro studies of RB200 (the first-generation pan-HER ligand trap) have focused on inhibition of cancer cell proliferation induced by lysophosphatidic acid (LPA) (21). LPA was chosen for these experiments because the GPCRs to which it binds (LPA receptor-1 [LPAR1], LPAR2, and LPAR3) couple with multiple ADAMs, resulting in release of ligands that activate both EGFR and HER3 (107). In addition, overexpression of LPAR occurs in multiple malignancies that are also associated with aberrant HER activation (75). RB200 demonstrated a broad range of activity in this assay (Table 1), indicating its potential for interrupting autocrine growth stimulation mediated by the GPCR/ADAM/HER family pathway (21).

Combinations of traditional therapeutics together with novel immunologic approaches will enable further general strategies that can simultaneously target the stromal and cancer cell components of a tumor. One possible direction is the engineering of 
immune effector cells (108) so that they target specific peptide fragments derived from HER family members overexpressed in cancer cells. Such peptides have been shown to be presented in the context of MHC class I at the cell surface of cancer cells (direct presentation) and at the cell surface of neighboring stromal cells (indirect or cross-presentation), creating a system in which both cell types can become targets for CTLs $(109,110)$. The process of antigen presentation by cancer and stromal cells is quite different from that of direct recognition of an epitope by a therapeutic $\mathrm{mAb}$ and is therefore likely to successfully target tumors that overexpress multiple HER family members, even if they have become resistant to the mAbs as a result of compensatory mechanisms. This is because expression of the targeted receptor (either EGFR or $\left.\mathrm{p} 185^{\mathrm{HER} 2}\right)$ is usually maintained in resistant cells in vitro and in patients $(11,12,66,92)$. Multiple independent studies have identified amino acids 369-377 of HER2 as a prominent HLA-A2.1restricted CTL epitope (originally identified by $\mathrm{T}$ cells of a patient with ovarian cancer) (111-113). Since identical epitopes are processed in humans and mice (114), possible side effects on somatic cells expressing the autochthonous mouse HER2 should be revealed in preclinical models, making them more relevant. The success of this approach depends on engineering a high-affinity TCR for the targeted epitope (115).

\section{Summary}

The HER family integrates stimuli originating from the serum, nonmalignant stromal cells, and the cancer cells themselves. We believe that this property of the HER family is likely to be an important reason for the remarkable success of cetuximab and trastuzumab and probably the TKIs that target EGFR and p185 HER2. Other properties of these therapeutics, such as their ability to mediate ADCC, may also contribute to their efficacy. Building on this success to achieve efficacy in a wider range of malignancies associated with aberrant activity of HER family members is the current challenge. Technologies described in this Review, which target both stromal and cancer cells, will be critical keys to success in meeting this challenge.

\section{Acknowledgments}

The authors gratefully acknowledge Axel Ullrich, Pei Jin, and John Huang for helpful discussions and Scott Patton for preparation of the manuscript and figures.

Address correspondence to: H. Michael Shepard, Receptor BioLogix, Inc., 3350 West Bayshore Road, Suite 150, Palo Alto, California 94303, USA. Phone: (650) 856-4617; Fax: (650) 856-4699; E-mail: hms@rblx.com.
1. DeNardo, D.G., and Coussens, L.M. 2007. Inflammation and breast cancer. Balancing immune response: crosstalk between adaptive and innate immune cells during breast cancer progression. Breast Cancer Res. 9:212.

2. Johansson, M., DeNardo, D.G., and Coussens, L.M. 2008. Polarized immune responses differentially regulate cancer development. Immunol. Rev. 222:145-154.

3. Tlsty, T.D., and Coussens, L.M. 2006. Tumor stroma and regulation of cancer development. Annu. Rev. Pathol. 1:119-150.

4. Hanahan, D., and Weinberg, R.A. 2000. The hallmarks of cancer. Cell. 100:57-70.

5. Nowell, P.C. 1986. Mechanisms of tumor progression. Cancer Res. 46:2203-2207.

6. Sporn, M.B., and Todaro, G.J. 1980. Autocrine secretion and malignant transformation of cells. N. Engl. J. Med. 303:878-880

7. Baselga,J. 2006. Targeting tyrosine kinases in cancer: the second wave. Science. 312:1175-1178.

8. Zhang, H., et al. 2007. ErbB receptors: from oncogenes to targeted cancer therapies. J. Clin. Invest. 117:2051-2058.

9. De Luca, A., et al. 2008. The role of the EGFR signaling in tumor microenvironment. J. Cell. Physiol. 214:559-567.

10. Wen, X.F., et al. 2006. HER2 signaling modulates the equilibrium between pro- and antiangiogenic factors via distinct pathways: implications for HER2-targeted antibody therapy. Oncogene. 25:6986-6996.

11. Ciardiello, F., and Tortora, G. 2008. EGFR antagonists in cancer treatment. N. Engl. J. Med. 358:1160-1174.

12. Hudis, C.A. 2007. Trastuzumab--mechanism of action and use in clinical practice. N. Engl. J. Med. 357:39-51.

13. Britsch, S. 2007. The neuregulin-I/ErbB signaling system in development and disease. Adv. Anat. Embryol. Cell Biol. 190:1-65.

14. Yarden, Y., and Sliwkowski, M.X. 2001. Untangling the ErbB signalling network. Nat. Rev. Mol. Cell Biol. 2:127-137.

15. Kuwai, T., et al. 2008. Phosphorylated epidermal growth factor receptor on tumor-associated endothelial cells is a primary target for therapy with tyrosine kinase inhibitors. Neoplasia. 10:489-500.

16. Bublil, E.M., and Yarden, Y. 2007. The EGF receptor family: spearheading a merger of signaling and therapeutics. Curr. Opin. Cell Biol. 19:124-134.

17. Mendelsohn, J., and Baselga, J. 2006. Epidermal growth factor receptor targeting in cancer. Semin. Oncol. 33:369-385.

18. Neve, R.M., Lane, H.A., and Hynes, N.E. 2001. The role of overexpressed HER2 in transformation. Ann. Oncol. 12:S9-S13.

19. Penuel, E., Akita, R.W., and Sliwkowski, M.X. 2002. Identification of a region within the ErbB2/ HER2 intracellular domain that is necessary for ligand-independent association. J. Biol. Chem. 277:28468-28473.

20. Cai, C.Q., et al. 2008. Epidermal growth factor receptor activation in prostate cancer by three novel missense mutations. Oncogene. 27:3201-3210.

21. Sarup, J., et al. 2008. Human EGF receptor (HER-1 HER-3) Fc-mediated heterodimer has broad antiproliferative activity in vitro and in human tumor xenografts. Mol. Cancer Ther. 7:3223-3236.

22. Revillion, F., Lhotellier, V., Hornez, L., Bonneterre, J., and Peyrat, J.P. 2008. ErbB/HER ligands in human breast cancer, and relationships with their receptors, the bio-pathological features and prognosis. Ann. Oncol. 19:73-80.

23. Stern, D.F. 2008. ERBB3/HER3 and ERBB2/HER2 duet in mammary development and breast cancer. J. Mammary Gland Biol. Neoplasia. 13:215-223.

24. Alimandi, M., et al. 1995. Cooperative signaling of ErbB3 and ErbB2 in neoplastic transformation and human mammary carcinomas. Oncogene. 10:1813-1821.

25. Normanno, N., et al. 2005. The ErbB receptors and their ligands in cancer: an overview. Curr. Drug Targets. 6:243-257.

26. Zhan, L., Xiang, B., and Muthuswamy, S.K. 2006 Controlled activation of ErbB1/ErbB2 heterodimers promote invasion of three-dimensional organized epithelia in an ErbB1-dependent manner: implications for progression of ErbB2-overexpressing tumors. Cancer Res. 66:5201-5208.

27. Gao, S.P., et al. 2007. Mutations in the EGFR kinase domain mediate STAT3 activation via IL-6 production in human lung adenocarcinomas. J. Clin. Invest. 117:3846-3856.
28. Schafer, Z.T., and Brugge, J.S. 2007. IL-6 involvement in epithelial cancers. J. Clin. Invest. 117:3660-3663.

29. Sasaki, T., et al. 2008. Modification of the primary tumor microenvironment by transforming growth factor alpha-epidermal growth factor receptor signaling promotes metastasis in an orthotopic colon cancer model. Am. J. Pathol. 173:205-216.

30. Weihua, Z., et al. 2008. Survival of cancer cells is maintained by EGFR independent of its kinase activity. Cancer Cell. 13:385-393.

31. Bianchi, S., et al. 2006. ErbB-receptors expression and survival in breast carcinoma: a 15 -year followup study. J. Cell. Physiol. 206:702-708.

32. Chow, N.H., Chan, S.H., Tzai, T.S., Ho, C.L., and Liu, H.S. 2001. Expression profiles of ErbB family receptors and prognosis in primary transitional cell carcinoma of the urinary bladder. Clin. Cancer Res. 7:1957-1962.

33. Koutsopoulos, A.V., et al. 2007. Simultaneous expression of c-erbB-1, c-erbB-2, c-erbB-3 and c-erbB-4 receptors in non-small-cell lung carcinomas: correlation with clinical outcome. Lung Cancer. 57:193-200.

34. Onn, A., et al. 2004. Synchronous overexpression of epidermal growth factor receptor and HER2-neu protein is a predictor of poor outcome in patients with stage I non-small cell lung cancer. Clin. Cancer Res. 10:136-143.

35. Wiseman, S.M., et al. 2005. Coexpression of the type 1 growth factor receptor family members HER-1, HER-2, and HER-3 has a synergistic negative prognostic effect on breast carcinoma survival. Cancer. 103:1770-1777.

36. Reiman, J.M., Kmieciak, M., Manjili, M.H., and Knutson, K.L. 2007. Tumor immunoediting and immunosculpting pathways to cancer progression. Semin. Cancer Biol. 17:275-287.

37. Finn, O.J. 2008. Cancer immunology. N. Engl. J. Med. 358:2704-2715.

38. Urban, J.L., Shepard, H.M., Rothstein, J.L., Sugarman, B.J., and Schreiber, H. 1986. Tumor necrosis factor: a potent effector molecule for tumor cell killing by activated macrophages. Proc. Natl. Acad. Sci.U.S. A. 83:5233-5237.

39. Sugarman, B.J., et al. 1985. Recombinant human tumor necrosis factor-alpha: effects on proliferation of normal and transformed cells in vitro. 
Science. 230:943-945.

40. Sugarman, B.J., Lewis, G.D., Eessalu, T.E., Aggarwal, B.B., and Shepard, H.M. 1987. Effects of growth factors on the antiproliferative activity of tumor necrosis factors. Cancer Res. 47:780-786.

41. Hudziak, R.M., Schlessinger, J., and Ullrich, A. 1987. Increased expression of the putative growth factor receptor $\mathrm{p} 185 \mathrm{HER} 2$ causes transformation and tumorigenesis of NIH $3 \mathrm{~T} 3$ cells. Proc. Natl. Acad. Sci. U. S. A. 84:7159-7163.

42. Perantoni, A.O., Rice, J.M., Reed, C.D., Watatani, M., and Wenk, M.L. 1987. Activated neu oncogene sequences in primary tumors of the peripheral nervous system induced in rats by transplacental exposure to ethylnitrosourea. Proc. Natl. Acad. Sci. U. S. A. 84:6317-6321.

43. Coussens, L., et al. 1985. Tyrosine kinase receptor with extensive homology to EGF receptor shares chromosomal location with neu oncogene. Science. 230:1132-1139.

44. Semba, K., Kamata, N., Toyoshima, K., and Yamamoto, T. 1985. A v-erbB-related protooncogene, c-erbB-2, is distinct from the c-erbB-1/epidermal growth factor-receptor gene and is amplified in a human salivary gland adenocarcinoma. Proc. Natl. Acad. Sci. U. S. A. 82:6497-6501.

45. King, C.R., Kraus, M.H., and Aaronson, S.A. 1985. Amplification of a novel v-erbB-related gene in a human mammary carcinoma. Science. 229:974-976.

46. McGuire, W.L. 1987. Prognostic factors for recurrence and survival in human breast cancer. Breast Cancer Res. Treat. 10:5-9.

47. Slamon, D.J., et al. 1987. Human breast cancer: correlation of relapse and survival with amplification of the HER-2/neu oncogene. Science. 235:177-182.

48. van de Vijver, M., et al. 1987. Amplification of the neu (c-erbB-2) oncogene in human mammary tumors is relatively frequent and is often accompanied by amplification of the linked c-erbA oncogene. Mol. Cell. Biol. 7:2019-2023.

49. Varley, J.M., Swallow, J.E., Brammar, W.J., Whittaker, J.L., and Walker, R.A. 1987. Alterations to either c-erbB-2(neu) or c-myc proto-oncogenes in breast carcinomas correlate with poor short-term prognosis. Oncogene. 1:423-430.

50. Hudziak, R.M., et al. 1988. Amplified expression of the HER2/ERBB2 oncogene induces resistance to tumor necrosis factor alpha in NIH 3T3 cells. Proc. Natl. Acad. Sci. U. S. A. 85:5102-5106.

51. Wang, X., Chen, W., and Lin, Y. 2007. Sensitization of TNF-induced cytotoxicity in lung cancer cells by concurrent suppression of the NF-kappaB and Akt pathways. Biochem. Biophys. Res. Commun. 355:807-812.

52. Zhou, B.P., et al. 2000. HER-2/neu blocks tumor necrosis factor-induced apoptosis via the Akt/NFkappaB pathway. J. Biol. Chem. 275:8027-8031.

53. Su, W.P., et al. 2007. HER-2/neu raises SHP-2, stops IFN-gamma anti-proliferation in bladder cancer. Biochem. Biophys. Res. Commun. 356:181-186.

54. Wilson, C.A., et al. 2005. HER-2 overexpression differentially alters transforming growth factor-beta responses in luminal versus mesenchymal human breast cancer cells. Breast Cancer Res. 7:R1058-R1079.

55. Fendly, B.M., et al. 1990. Characterization of murine monoclonal antibodies reactive to either the human epidermal growth factor receptor or HER2/neu gene product. Cancer Res. 50:1550-1558.

56. Lewis, G.D., et al. 1993. Differential responses of human tumor cell lines to anti-p185HER2 monoclonal antibodies. Cancer Immunol. Immunother. 37:255-263

57. Hudziak, R.M., et al. 1989. p185HER2 monoclonal antibody has antiproliferative effects in vitro and sensitizes human breast tumor cells to tumor necrosis factor. Mol. Cell. Biol. 9:1165-1172.

58. Shepard, H.M., et al. 1991. Monoclonal antibody therapy of human cancer: taking the HER2 proto- oncogene to the clinic. J. Clin. Immunol. 11:117-127.

59. Hudziak, R.M., Lewis, G.D., Holmes, W.E., Ullrich, A., and Shepard, H.M. 1990. Selection for transformation and met protooncogene amplification in NIH 3T3 fibroblasts using tumor necrosis factor alpha. Cell Growth Differ. 1:129-134.

60. Carter, P., et al. 1992. Humanization of an antip185HER2 antibody for human cancer therapy. Proc. Natl. Acad. Sci. U. S. A. 89:4285-4289.

61. Friedman, L.M., et al. 2005. Synergistic down-regulation of receptor tyrosine kinases by combinations of mAbs: implications for cancer immunotherapy. Proc. Natl. Acad. Sci. U. S. A. 102:1915-1920.

62. Nagy, P., et al. 1999. Activation-dependent clustering of the erbB2 receptor tyrosine kinase detected by scanning near-field optical microscopy. J. Cell Sci. 112:1733-1741.

63. Yang, S., et al. 2007. Mapping ErbB receptors on breast cancer cell membranes during signal transduction. J. Cell Sci. 120:2763-2773.

64. Varchetta, S., et al. 2007. Elements related to heterogeneity of antibody-dependent cell cytotoxicity in patients under trastuzumab therapy for primary operable breast cancer overexpressing Her2. Cancer Res. 67:11991-11999.

65. Musolino, A., et al. 2008. Immunoglobulin G fragment $C$ receptor polymorphisms and clinical efficacy of trastuzumab-based therapy in patients with HER-2/neu-positive metastatic breast cancer. J. Clin. Oncol. 26:1789-1796.

66. Ritter, C.A., et al. 2007. Human breast cancer cells selected for resistance to trastuzumab in vivo overexpress epidermal growth factor receptor and ErbB ligands and remain dependent on the ErbB receptor network. Clin. Cancer Res. 13:4909-4919.

67. Barok, M., et al. 2008. Trastuzumab decreases the number of circulating and disseminated tumor cells despite trastuzumab resistance of the primary tumor. Cancer Lett. 260:198-208.

68. Li, Y.M., et al. 2004. Upregulation of CXCR4 is essential for HER2-mediated tumor metastasis. Cancer Cell. 6:459-469.

69. Britten, C.D. 2004. Targeting ErbB receptor signaling: a pan-ErbB approach to cancer. Mol. Cancer Ther. 3:1335-1342.

70. Hynes, N.E., Horsch, K., Olayioye, M.A., and Badache, A. 2001. The ErbB receptor tyrosine family as signal integrators. Endocr. Relat. Cancer. 8:151-159.

71. Daub, H., Weiss, F.U., Wallasch, C., and Ullrich, A. 1996. Role of transactivation of the EGF receptor in signalling by G-protein-coupled receptors. Nature. 379:557-560

72. Karnoub, A.E., and Weinberg, R.A. 2006. Chemokine networks and breast cancer metastasis. Breast Dis. 26:75-85.

73. Rozengurt, E. 2007. Mitogenic signaling pathways induced by $\mathrm{G}$ protein-coupled receptors. J. Cell. Physiol. 213:589-602.

74. Luppi, F., Longo, A.M., de Boer, W.I., Rabe, K.F., and Hiemstra, P.S. 2007. Interleukin-8 stimulates cell proliferation in non-small cell lung cancer through epidermal growth factor receptor transactivation. Lung Cancer. 56:25-33.

75. Murph, M., and Mills, G.B. 2007. Targeting the lipids LPA and S1P and their signalling pathways to inhibit tumour progression. Expert Rev. Mol. Med. 9:1-18.

76. Pastore, S., Mascia, F., Mariani, V., and Girolomoni, G. 2008. The epidermal growth factor receptor system in skin repair and inflammation. J. Invest. Dermatol. 128:1365-1374.

77. Pattarozzi, A., et al. 2008. 17beta-estradiol promotes breast cancer cell proliferation-inducing stromal cell-derived factor-1-mediated epidermal growth factor receptor transactivation: reversal by gefitinib pretreatment. Mol. Pharmacol. 73:191-202.

78. Ohtsu, H., Dempsey, P.J., and Eguchi, S. 2006.
ADAMs as mediators of EGF receptor transactivation by $\mathrm{G}$ protein-coupled receptors. Am. J. Physiol. Cell Physiol. 291:C1-C10.

79. Argast, G.M., Campbell, J.S., Brooling, J.T., and Fausto, N. 2004. Epidermal growth factor receptor transactivation mediates tumor necrosis factor-induced hepatocyte replication. J. Biol. Chem. 279:34530-34536.

80. Chen, W.N., et al. 2004. Induced autocrine signaling through the epidermal growth factor receptor contributes to the response of mammary epithelial cells to tumor necrosis factor alpha. J. Biol. Chem. 279:18488-18496.

81. Joo, C.K., et al. 2008. Ligand release-independent transactivation of epidermal growth factor receptor by transforming growth factor-beta involves multiple signaling pathways. Oncogene. 27:614-628.

82. Evdonin, A.L., Guzhova, I.V., Margulis, B.A., and Medvedeva, N.D. 2006. Extracellular heat shock protein 70 mediates heat stress-induced epidermal growth factor receptor transactivation in A431 carcinoma cells. FEBS Lett. 580:6674-6678.

83. Martin, M.U., and Wesche, H. 2002. Summary and comparison of the signaling mechanisms of the Toll/interleukin-1 receptor family. Biochim. Biophys. Acta. 1592:265-280.

84. Hallbeck, A.L., Walz, T.M., Briheim, K., and Wasteson, A. 2005. TGF-alpha and ErbB2 production in synovial joint tissue: increased expression in arthritic joints. Scand. J. Rheumatol. 34:204-211.

85. Donepudi, M., and Resh, M.D. 2008. c-Src trafficking and co-localization with the EGF receptor promotes EGF ligand-independent EGF receptor activation and signaling. Cell Signal. 20:1359-1367.

86. Arora, P., Cuevas, B.D., Russo, A., Johnson, G.L., and Trejo, J. 2008. Persistent transactivation of EGFR and ErbB2/HER2 by protease-activated receptor-1 promotes breast carcinoma cell invasion. Oncogene. 27:4434-4445.

87. Chinni, S.R., et al. 2008. CXCL12/CXCR4 transactivates HER2 in lipid rafts of prostate cancer cells and promotes growth of metastatic deposits in bone. Mol. Cancer Res. 6:446-457.

88. Walters, D.K., and Jelinek, D.F. 2004. A role for Janus kinases in crosstalk between ErbB 3 and the interferon-alpha signaling complex in myeloma cells. Oncogene. 23:1197-1205.

89. Croce, C.M. 2008. Oncogenes and cancer. N. Engl. J. Med. 358:502-511.

90. Adams, C.W., et al. 2006. Humanization of a recombinant monoclonal antibody to produce a therapeutic HER dimerization inhibitor, pertuzumab. Cancer Immunol. Immunother. 55:717-727.

91. Hudelist, G., et al. 2003. Co-expression of ErbBfamily members in human breast cancer: Her$2 /$ neu is the preferred dimerization candidate in nodal-positive tumors. Breast Cancer Res. Treat. 80:353-361.

92. Wheeler, D.L., et al. 2008. Mechanisms of acquired resistance to cetuximab: role of HER (ErbB) family members. Oncogene. 27:3944-3956.

93. Cai, Z., et al. 2008. Differential binding patterns of monoclonal antibody 2C4 to the ErbB3-p185 (her2/neu) and the EGFR-p185 (her2/neu) complexes. Oncogene. 27:3870-3874.

94. Motoyama, A.B., Hynes, N.E., and Lane, H.A. 2002. The efficacy of ErbB receptor-targeted anticancer therapeutics is influenced by the availability of epidermal growth factor-related peptides. Cancer Res. 62:3151-3158.

95. Sergina, N.V., et al. 2007. Escape from HER-family tyrosine kinase inhibitor therapy by the kinaseinactive HER3. Nature. 445:437-441.

96. Arpino, G., et al. 2007. Treatment of human epidermal growth factor receptor 2-overexpressing breast cancer xenografts with multiagent HER-targeted therapy. J. Natl. Cancer Inst. 99:694-705.

97. Franklin, M.C., et al. 2004. Insights into ErbB sig- 
naling from the structure of the ErbB2-pertuzumab complex. Cancer Cell. 5:317-328.

98. Huang, G., Chantry, A., and Epstein, R.J. 1999. Overexpression of ErbB2 impairs ligand-dependent downregulation of epidermal growth factor receptors via a post-transcriptional mechanism. J. Cell. Biochem. 74:23-30.

99. Burstein, H.J., et al. 2008. A phase II study of lapatinib monotherapy in chemotherapy-refractory HER2-positive and HER2-negative advanced or metastatic breast cancer. Ann. Oncol. 19:1068-1074.

100. Herbst, R.S., et al. 2007. Efficacy and safety of single-agent pertuzumab, a human epidermal receptor dimerization inhibitor, in patients with non small cell lung cancer. Clin. Cancer Res. 13:6175-6181.

101. Hynes, N.E. 2007. Targeting ERBB receptors in cancer. Recent Results Cancer Res. 172:45-57.

102.Reid, A., Vidal, L., Shaw, H., and de Bono, J. 2007. Dual inhibition of ErbB1 (EGFR/HER1) and ErbB2 (HER2/neu). Eur. J. Cancer. 43:481-489.

103.Fridman, J.S., et al. 2007. Selective inhibition of ADAM metalloproteases as a novel approach for modulating ErbB pathways in cancer. Clin. Cancer Res. 13:1892-1902.

104. Fisher, J.F., and Mobashery, S. 2006. Recent advances in MMP inhibitor design. Cancer Metastasis Rev.
25:115-136.

105.Rocks, N., et al. 2008. Emerging roles of ADAM and ADAMTS metalloproteinases in cancer. Biochimie. 90:369-379.

106.Tracey, D., Klareskog, L., Sasso, E.H., Salfeld, J.G., and Tak, P.P. 2008. Tumor necrosis factor antagonist mechanisms of action: a comprehensive review. Pharmacol. Ther. 117:244-279.

107. Higashiyama, S., et al. 2008. Membrane-anchored growth factors, the epidermal growth factor family: beyond receptor ligands. Cancer Sci. 99:214-220.

108. Richman, S.A., et al. 2006. Development of a novel strategy for engineering high-affinity proteins by yeast display. Protein Eng. Des. Sel. 19:255-264.

109.Zhang, B., Karrison, T., Rowley, D.A., and Schreiber, H. 2008. IFN-g- and TNF-dependent bystander eradication of antigen-loss variants in established mouse cancers. J. Clin. Invest. 118:1398-1404.

110.Zhang, B., et al. 2008. Equilibrium between host and cancer caused by effector $\mathrm{T}$ cells killing tumor stroma. Cancer Res. 68:1563-1571.

111.Fisk, B., Blevins, T.L., Wharton, J.T., and Ioannides, C.G. 1995. Identification of an immunodominant peptide of HER-2/neu protooncogene recognized by ovarian tumor-specific cytotoxic Tlymphocyte lines. J. Exp. Med. 181:2109-2117.
112.Keogh, E., et al. 2001. Identification of new epitopes from four different tumor-associated antigens: recognition of naturally processed epitopes correlates with HLA-A*0201-binding affinity. J. Immunol. 167:787-796

113.Lustgarten, J., et al. 1997. Identification of Her-2/ Neu CTL epitopes using double transgenic mice expressing HLA-A2.1 and human CD.8. Hum. Immunol. 52:109-118.

114.Lustgarten, J., Dominguez, A.L., and Cuadros, C. 2004. The CD8+ T cell repertoire against Her-2/neu antigens in neu transgenic mice is of low avidity with antitumor activity. Eur. J. Immunol. 34:752-761.

115.Richman, S.A., and Kranz, D.M. 2007. Display, engineering, and applications of antigen-specific $\mathrm{T}$ cell receptors. Biomol. Eng. 24:361-373.

116.Schlange, T., Matsuda, Y., Lienhard, S., Huber, A., and Hynes, N.E. 2007. Autocrine WNT signaling contributes to breast cancer cell proliferation via the canonical WNT pathway and EGFR transactivation. Breast Cancer Res. 9:R63.

117. Prenzel, N., Zwick, E., Leserer, M., and Ullrich, A. 2000. Tyrosine kinase signalling in breast cancer. Epidermal growth factor receptor: convergence point for signal integration and diversification. Breast Cancer Res. 2:184-190. 